\title{
THE INTERNAL STRUCTURE OF THE SUN AND
}

\section{SOLAR TYPE STARS}

\author{
IAN W. ROXBURGH \\ Queen Mary College, University of London \\ and \\ High Altitude Observatory, National Centre for Atmospheric Research, Boulder, Colo., U.S.A.
}

\begin{abstract}
Our understanding of the internal structure of the Sun and solar type stars has been undermined by recent observations. In this paper we consider some of the puzzles and possible resolutions; the solar neutrino problem, lithium and beryllium abundance, rotation and calcium emission, variation of the solar constant, solar oscillations and the solar convective zone. The picture that emerges is one of confusion, and so it should be since we have no idea of what is going on inside the Sun and a fortiori of what is going on inside other stars.
\end{abstract}

\section{Introduction}

A few years ago, the internal structure and evolution of stars was thought to be well understood. The theory could more or less explain the few observations that existed and there was every confidence that other details would also be explained within the same theoretical structure.

This dream world of the stellar theoretician is now ended. The stellar theorist who had only a passing interest in the Sun has been, is being, or should be, forced to re-examine his theoretical speculations in the light of new data from the Sun. Solar physics has forced the stellar physicist to think again, and the results of that re-thinking could have repercussions in many branches of astrophysics and perhaps other branches of physics as well.

Perhaps the most spectacular experiments are the abortive search for solar neutrinos by Davis and co-workers (Davis 1972), measurements of solar oblateness by Dicke and Goldenberg (1967) and the improved measurements by Henry Hill and his co-workers, with their measurements of oscillations of the Sun (Hill et al., 1975). But other observations have added to the confusion; abundance measurements of lithium and beryllium in the Sun and other stars, (Wallerstein and Conti, 1969) observations of $\mathrm{Ca}^{+}$emission in the Sun and stars (Wilson, 1963), measurements of the solar wind, geological evidence from Earth, and theoretical work on climate modelling (Hays, 1975). The observations of multi-scale convection on the Sun has raised doubts about the mixing length theory and when all placed together, it is difficult to escape the conclusion that something is wrong with standard ideas on sfellar structure. While we have not yet evolved a new theory, the need to accommodate these new results has made the study of the internal structure of the Sun and solar type stars one of the most exciting and challenging areas of research.

In this paper I want to describe some of these dilemmas and proposed resolutions. I cannot give you a new theory for the solar interior for one does not exist - all is confusion. So in contrast to most papers, the purposes of this paper is to confuse you, not to enlighten you. This should be an easy task for one whose papers are normally confusing, even when they are not supposed to be. 


\section{A Simple Man's Guide to the Internal Structure of the Sun}

The standard models of stellar structure can be found in many textbooks (e.g. Schwarzschild, 1958). In arriving at these models many assumptions are made which we now have to question; let me list some of them:

(1) The stars are in hydrostatic equilibrium with pressure balancing gravity - there are no significant accelerations.

(2) The stars are in thermal equilibrium - the energy radiated is equal to the energy produced so there are no secular changes, except in the early pre-main sequence contraction phase of solar evolution.

(3) Energy is carried by radiation only in those regions of a star which are stable against convection according to the Schwarzschild criterion - there is no significant transport of energy by waves.

(4) The energy produced is due to nuclear burning of hydrogen to helium.

(5) The only regions where matter is chemically mixed are those regions where convection takes place.

(6) Convective regions can be adequately modelled by the mixing length theory of convection, which is a purely local theory. Thus convective overshooting is considered to be unimportant.

(7) Mass loss, rotation and magnetic fields are unimportant and can be treated as a small perturbation to the standard model.

(8) The Sun was initially homogeneous in composition and its age is the same as the Earth, approximately $4.7 \times 10^{9} \mathrm{yr}$.

(9) The laws of physics are time independent.

With these assumptions, models of solar type (and other) stars can be constructed. They have had moderate success; it is possible to construct a model of the present Sun that agrees with its two main characteristics, the present luminosity and radius of the Sun.

But such agreement with observation is not very significant: the models of the Sun have two degrees of freedom, the helium abundance and the mixing length in the theory of convection. The initial helium abundance is adjusted to obtain the correct luminosity and the mixing length to get the correct radius; the agreement between prediction and observation is, therefore, no check at all! As we shall see, the classical models constructed in this way make other predictions, which at least for the Sun, can be tested at various levels of accuracy - the models do not fare so well!

The classical picture that emerges is that the Sun was formed from the collapse of a gas cloud. The collapse stopped when the matter became ionised and the proto sun had a radius of about $50 R_{\odot}$, a central temperature of maybe $50000 \mathrm{~K}$ and an effective temperature of about $3500 \mathrm{~K}$. During the Hayashi phase (Hayashi, 1961), the proto sun was fully convective from centre to surface and very luminous, the energy radiated into space causing the star to contract releasing gravitational energy and increasing its internal temperature. As the contraction continues, the convection dies away in a growing region around the centre and the temperature in the mixed convective region never exceeds about $1.5 \times 10^{6} \mathrm{~K}$. Eventually, the central regions become hot enough for nuclear reactions to be ignited, this energy release compensates for the energy loss by radiation and the gravitational contraction ceases. 
Future evolution is now due to the hydrogen burning in the centre. This increases the helium abundance in the central regions (which are unmixed) and the nonuniform chemical composition produces slow changes in the Sun's internal structure until after $4.7 \times 10^{9} \mathrm{yr}$, it has the present radius and luminosity.

These models predict a number of consequences that can now be subjected to some tests.

(1) Present central temperature of the $\operatorname{Sun} \simeq 1.5 \times 10^{7} \mathrm{~K}$-neutrino flux $\simeq$ $6 \mathrm{SNU}^{*}$.

(2) Luminosity of the Sun has monotonically increased from 0.65 to $1 L_{\odot}$ over the last $4 \times 10^{9} \mathrm{yr}$.

(3) The matter in the surface layers has never been at temperatures greater than $1.5 \times 10^{6} \mathrm{~K}$.

(4) The Sun is stable to oscillations.

In the next sections, I want to discuss the contradiction between these predictions and the observations and what we can conclude from them. Put briefly, there is evidence that all four predictions are wrong, since

(1) The upper limit on the neutrino flux is 1 SNU.

(2) The paleoclimatological evidence, and theoretical climate modelling indicates $L \simeq(1 \pm 0.05) L_{\odot}$ over $4 \times 10^{9} \mathrm{yr}$.

(3) The lithium abundance in the surface layers is very low indicating that the matter in the surface layers has been mixed to about $3 \times 10^{6} \mathrm{~K}$.

(4) Recent observations by Hill indicate that the Sun is oscillating in its normal modes.

One hope for the orthodox point of view is that the inclusion of neglected effects like rotation and magnetic fields can save the models; the heterodox view which I wish to urge on you is that the models cannot be saved - we need to reconsider our basic physical assumptions. We can make a preliminary guess at the internal structure of the Sun. It could have:

(1) Present central temperature approx $1.2 \times 10^{7} \mathrm{~K}$.

(2) A large central region that is chemically mixed on a time scale less than $10^{9} \mathrm{yr}$.

(3) Some mixing process that carries surface layer material down to a temperature of $3 \times 10^{6} \mathrm{~K}$ but not to $4 \times 10^{6} \mathrm{~K}$ since beryllium has not been burnt - this could be substantial convective overshooting.

(4) Some mechanism for exciting oscillations - a convective core or some other central instability.

\section{The Solar Neutrino Problem}

The nuclear reactions in the proton-proton chain that are thought to be responsible for energy production have many branches. The neutrinos that should be detected are produced in the boron-beryllium reaction ${ }^{8} \mathrm{~B} \rightarrow{ }^{8} \mathrm{Be}+\mathrm{e}^{+}+\nu$; although only a small fraction of the reactions are along this chain (about $0.02 \%$ ) this is the important branch for the production of neutrinos. The fraction of reactions on each branch of

* $1 \mathrm{SNU}=10^{-36}$ captures per ${ }^{37} \mathrm{Cl}$ atom per second. 
the p-p chain is sensitive to the temperature. At high temperature a large fraction is on the boron-beryllium branch and at low temperatures a smaller fraction: thus the neutrino flux is a sensitive thermometer giving an estimate of the central temperature of the Sun. For a flux of $1 \mathrm{SNU}$, the temperature has to be about $12-13 \times 10^{7} \mathrm{~K}$.

The cross-section for the absorption of neutrinos is very small and thus almost all the neutrinos escape from the Sun without being absorbed. In neutrinos we are therefore looking at the Sun now (or rather $8 \mathrm{~min}$ ago). This is to be contrasted with photon measurements: photons are readily absorbed and the photon diffusion time through the Sun is of the order of $3 \times 10^{7} \mathrm{yr}$. Thus one possibility that has been advocated (Fowler, 1972; Dilke and Gough, 1973) is that the centre of the Sun is indeed at the temperature suggested by the neutrino experiment and is, therefore, too cool to produce much energy - there is a thermal inbalance which will manifest itself over the thermal diffusion time scale of $3 \times 10^{7} \mathrm{yr}$. Before examining this proposal, let us consider a number of other proposed resolutions.

\subsection{Homogeneous Sun}

If the Sun has been mixed throughout its lifetime, the chemical composition is still uniform and the hydrogen abundance in the centre is larger than in the standard model - there is more fuel available and therefore it burns at a slower rate - that is, at a lower temperature. This can lower the neutrino flux to 1.5-2 SNU, still above the experimental limit. However, if the Sun were mixed, all the beryllium in the surface layers would be burnt - which it is not. The smaller the fraction of matter that is mixed, the higher the neutrino flux. However, a mixed model gives only a very small change in the Sun's luminosity over its lifetime.

\subsection{RAPIDLY SPINNING CORE OR LARGE CENTRAL MAGNETIC FIELD}

If there is some other contribution to the support of the Sun than pressure, then it is possible to affect the neutrino flux. In order to lower this flux, but still produce the luminosity observed, then the energy must be produced at a lower temperature over a larger region. This will happen if the ratio of the extra supporting force (centrifugal or magnetic) to gravity decreases away from the centre (Roxburgh, 1974, 1975a). However, such models give a substantial quadrupole moment at variance with the most recent determinations by Hill and co-workers (Hill and Stebbins, 1975). There are other objections: would such a distribution of angular momentum or magnetic flux be stable? The answer is almost certainly no, the magnetic flux would float and the rotation would be unstable to thermal instabilities (e.g. Goldreich and Schubert, 1967; Fricke, 1968). Perhaps a chemical composition gradient could stabilise the rotation, but it is difficult to build a cosmogonical picture of why the centre should spin as rapidly as required, yet have a small quadrupole moment (Roxburgh, 1974). Moreover, if the central region contains any magnetic field, this would link regions of different angular velocity and wipe out the required large inward increase in spin. A large magnetic field, even if stable, would decay on a time scale of order $10^{9} \mathrm{yr}$ leaving only the fewest slowly decaying modes which are nowhere near sufficiently centrally condensed to lower the neutrino flux. 


\subsection{VARYING CONSTANT OF GRAVITY}

Various attempts to incorporate Mach's Principle into a theory of gravitation and dynamics predict that the 'constant' of gravity $G$ changes as the Universe expands. Some models (Milne, 1935) give $G \propto t$, others (Dirac, 1936) give $G \propto 1 / t$. The effect of such a change is to lower the neutrino flux to $2 \mathrm{SNU}$ in the Milne case (Roxburgh, 1973) and to increase it to $20 \mathrm{SNU}$ in the Dirac case (Shaviv and Bachall, 1969). In both cases the luminosity of the Sun varies significantly since $L \propto G^{7}$ for the Sun, and even though the diameter of the Earth's orbit changes as $G$ changes, $d \propto 1 / G$, the flux of radiation falling on the Earth changes by factors of 4 or more. This is difficult to reconcile with the paleoclimatological evidence that the flux has been remarkably constant over the age of the Earth.

\subsection{Chemical anomalies}

If the central composition of the Sun is significantly different from the surface layers, in particular, if it has a very high heavy element abundance, $Z$, then the structure is significantly changed. A recent model by Hoyle (1975) has a core of $0.5 M_{\odot}$ with $Z \sim 0.5$, which because of the high opacity is convective, and an envelope of $0.5 M_{\odot}$ and normal composition $Z \sim 0.02$. This model gives a low neutrino flux of less than 1 SNU, it also predicts an almost constant solar luminosity. However, it is difficult to understand the cosmogonical history of this model. Hoyle suggests that the inner half of the Sun was formed first from heavy element rich matter produced in a fluctuation in the early stages of the Universe; the rest of the matter was added later some $4.7 \times 10^{9} \mathrm{yr}$ ago - but how? Until some plausible scheme for the formation of such a two zone Sun is put forward, this model is highly suspect.

\subsection{WAVE TRANSPORT OF ENERGY}

Recently both Hoyle (1975) and Hill (1975) have suggested that a significant fraction of the Sun's energy is transported by waves rather than radiation: this would change the structure of the Sun and could possibly lower the central temperature and hence the neutrino flux. No detailed models have been constructed, so it is difficult to say whether this would be significant, indeed, since the Sun has to produce $1 L_{\odot}$, wave transport is not necessarily helpful. It is necessary to change the temperature profile in the centre of the Sun in a manner similar to the change produced by a very rapidly spinning core, and it has not been shown that this is possible.

\subsection{Periodic instabilities ANd thermal inbalanCe}

It was pointed out by Gough and Dilke that the Sun is possibly unstable to non radial oscillations during its evolution. At the high temperature in the central regions of the Sun, the proton-proton fusion chain goes to completion to ${ }^{4} \mathrm{He}$, but at lower temperatures away from the centre the reactions only proceed to ${ }^{3} \mathrm{He}$. After some time, $\mathrm{a}^{3} \mathrm{He}$ concentration develops away from the centre and this is potentially an unstable situation. If such ${ }^{3} \mathrm{He}$ rich matter is displaced to regions of higher temperature, it rapidly burns to ${ }^{4} \mathrm{He}$ the energy released enhancing the displacement. Detailed calculations by Christian Dalsgaard et al. (1974) show that such an 
instability sets in very early in the Sun's evolution, after some $2 \times 10^{8} \mathrm{yr}$ from the beginning of nuclear burning. The growth rate of the instability is long, and what eventually happens is speculation, but the suggestion is that the central regions are mixed, increasing the ${ }^{3} \mathrm{He}$ burning. The Sun is then out of thermal balance and readjusts on a thermal time scale, the ${ }^{3} \mathrm{He}$ then relaxes to equilibrium and the central temperature is low. The neutrino flux during this stage is also low until the Sun has adjusted again to thermal equilibrium and in a further $2 \times 10^{8} \mathrm{yr}$ the process repeats. In this model we just happen to be observing in a period of anomalously low neutrino flux.

At present there are no adequate models of the evolution of the Sun with such a repetitive instability, nor are the consequences of such in instability known. Preliminary, though inappropriate calculations by Ulrich, suggest that a large variation in the Sun's luminosity during the mixing process $(\simeq 40 \%)$ but again it is premature to judge this model. It is certainly very promising; if the inner $40-50 \%$ of the Sun was mixed every $2-3 \times 10^{8} \mathrm{yr}$, the average luminosity of the Sun would change very little. However, paleoclimatological research suggests that short time variations of greater than $5 \%$ in the Sun's luminosity have not occurred; could it be that the ${ }^{3} \mathrm{He}$ instability drives steady currents slowly mixing the interior? It is worth remarking that if the inner half of stars like the Sun are effectively mixed on a time scale shorter than the evolution time scale, it will significantly affect the time scale and characteristics of stellar evolution. This in turn will affect the estimates of the age of globular clusters, the evolution of galaxies and therefore the correction for galactic evolution in cosmological investigations.

\section{Variation of the Solar Constant}

While paleoclimatology is still in a very early stage of development, it is worth reporting the tentative conclusions that have been reached so far. The pertinent observations are (a) that the Earth is not covered with ice at the present time! (b) the geological record indicates that the Earth has never been completely covered with ice and (c) that life in some form has existed for as long as $3 \times 10^{9} \mathrm{yr}$. The climate models on the other hand indicate that if the Sun's luminosity was less than $95 \%$ of its present value, the Earth would be iced over and indeed that it would then remain ice covered if the luminosity increased to its present value, since an ice covered Earth would have a much higher albedo and therefore reflect a large part of the incoming radiation.

Moreover, while it is tempting to identify the ice ages with variations in the solar luminosity, the current climate models indicate that these correspond to at most a $3 \%$ variation in the solar constant, but can anyway be explained by plate motion and continental wandering on the Earth's surface without any variation in the Sun's luminosity. While it is perhaps premature to put too much weight on these results, the present conclusions are (Hays, 1975):

$$
\begin{aligned}
& L=(1 \pm 0.05) L_{\odot} \quad t \simeq 3 \times 10^{9} \mathrm{yr} \\
& \Delta L / L \leqq 0.03 \text { on time scales } t \simeq 3.10^{8} \mathrm{yr} .
\end{aligned}
$$


These results are difficult to reconcile with current models of the Sun and solar type stars. In the standard evolutionary models the Sun's luminosity has increased monotonically from about $0.6 L_{\odot}$ to $1 L_{\odot}$ over its lifetime of $4.7 \times 10^{9} \mathrm{yr}$, and most of this variation has been over the last $3 \times 10^{9} \mathrm{yr}$. If the Sun's luminosity is to vary by less than $5 \%$, then a substantial region in the centre of the Sun has to be mixed on a time scale of less than $10^{9} \mathrm{yr}$; the mixing does not have to be fast (as in a convective core) and periodic mixing on a time scale of $3 \times 10^{8} \mathrm{yr}$ is compatible with the climate models provided that during this mixing the Sun's luminosity does not vary by more than $3 \%$. We could, therefore, imagine an evolutionary pattern similar to that suggested by Dilke and Gough (1973); an inhomogeneous evolution until the onset of an instability which mixes the central regions of the Sun, and the pattern repeats. The problem with this mode of evolution is that it seems to indicate a large change in the solar constant during the readjustment period (Ulrich, 1975), so again we are led to suggest a slow steady mixing in the solar interior perhaps a sort of semi-convection keeping the Sun on the verge of stability, lowering the neutrino flux and keeping the solar constant constant.

\section{Lithium and Beryllium Abundance, Rotation and Calcium Emission}

Turning now to the surface layers of solar type stars, there are other enigmas. The Sun is very low in lithium but normal in beryllium abundance; since lithium burns at about $3 \times 10^{6} \mathrm{~K}$ and beryllium at $4 \times 10^{6} \mathrm{~K}$, this indicates that the matter in the outer regions of the Sun has at some time been at temperatures between these limits. The standard solar evolutionary models predict that the matter in the outer layers has never been hotter than $2 \times 10^{6} \mathrm{~K}$.

One possible explanation could be that the Sun has lost a lot of mass due to enhanced activity at an early stage in its lifetime; if, say, the Sun has lost $20 \%$ of its mass since it was near the main sequence, then the matter that is now in the convective zone was $\frac{4}{5}$ of the way (by mass) from the centre of the original Sun of mass $1.25 M_{\odot}$ and therefore at a temperature of about $4 \times 10^{6} \mathrm{~K}$. This matter could, therefore, be depleted in lithium but not beryllium.

Another possibility is that the convective motions in the outer envelope of the Sun penetrates well inside the stable regions so carrying down to regions where lithium could be burnt, or the convective zone itself could be very much deeper than current models predict (see Section 7). Indeed, recent calculations I have made suggest that the simple mixing length theory is basically incorrect and has to be replaced by a non local theory of turbulent convection which requires substantial overshooting to reduce the convective velocities to zero (Roxburgh, 1975).

But one of the possible clues to the lithium-beryllium problem may be in the correlation between lithium abundance, rotation and calcium emission pointed out by Skumanich (1972); all seem to decrease with age on a time scale of the order of $10^{9} \mathrm{yr}$, although the present solar lithium abundance is anomalously low. The picture suggested by Skumanich (1972) and Durney (1972) is that the $\mathrm{Ca}^{+}$emission is an indicator of magnetic field strength $B$, and that due to angular momentum loss in the solar wind, the Sun's rotation is gradually decreased. This decrease in the rotation 
results in a lower magnetic field strength due to a reduced dynamo action since the dynamo field $B$ is conjectured to be proportional to the angular velocity $\Omega$. The rate of change of angular momentum $H$ is then:

$$
\frac{d H}{\mathrm{~d} t} \propto \frac{\mathrm{d} M}{\mathrm{~d} t} \Omega r_{\mathrm{A}}^{2}
$$

the mass loss

$$
\frac{\mathrm{d} M}{\mathrm{~d} t}=4 \pi \rho_{\mathrm{A}} u_{\mathrm{A}} r_{\mathrm{A}}^{2}
$$

where $r_{\mathrm{A}}$, the radial distance at which the flow goes Alfénic is given by

$$
4 \pi \rho u_{\mathrm{A}}^{2}=B_{\mathrm{A}}^{2}
$$

hence

$$
\frac{\mathrm{d} H}{\mathrm{~d} t} \propto \frac{\Omega B_{\mathrm{A}}^{2} r_{\mathrm{A}}^{4}}{u_{\mathrm{A}}} \propto \frac{\Omega B_{\odot}^{2} R^{4}}{u_{\mathrm{A}}}
$$

since $\mathrm{Br}^{2}$ is constant for a radial field. If the solar wind has remained unchanged (a dubious assumption since the coronal heating and resulting solar wind is likely to be changed by the changing solar magnetic field) and is therefore constant so

$$
\frac{\mathrm{d} H}{\mathrm{~d} t} \propto \Omega B_{\odot}^{2} \propto \Omega^{3}
$$

if the dynamo gives $B \propto \Omega$. The solution of this equation gives approximately

$$
\left(\frac{\Omega}{\Omega_{\odot}}\right)^{2}=\frac{5 \times 10^{9}}{t}
$$

which gives the present angular velocity for the Sun, consistent with the Sun rotating approximately uniformly throughout.

This slowing down of the Sun by the magnetic solar wind has been used by Dicke (1972) to explain the lithium-beryllium problem. The wind slows down the surface layers first which being strongly coupled to the convective zone slows down the outer convective zone. This produces a layer of differential rotation beneath the solar convective zone in which slow thermally driven turbulence couples the outer zone down to a depth where lithium is depleted.

The thermally driven turbulence is due to the instability of differential rotation first pointed out by Goldreich and Schubert (1967) and Fricke (1968). A rotating liquid is dynamically unstable if the angular momentum per unit mass decreases outwards (Rayleigh Instability)

$$
\frac{\partial}{\partial \varpi}\left(\Omega \varpi^{2}\right)<0
$$

That this instability is also operative in stably stratified layers was noticed by myself (Roxburgh, 1964) since there will always exist a displacement for which the stabilising effect is negligible, but Goldreich, Schubert and Fricke showed that the 
Taylor-Proudman theorem had also to be satisfied, namely

$$
\frac{\partial \Omega}{\partial z}=0
$$

$(\varpi, \varphi, z)$ being cylindrical polar coordinates, $\varpi=0$ the axis of rotation. Moreover, their detailed stability analysis showed that these results are only strictly true for zero Prandtl number $\sigma=\nu / \kappa$. For the Sun $\sigma \simeq 10^{-6}$ so there can exist a stable angular velocity gradient beneath the solar convective zone, with

$$
\frac{\mathrm{d} \Omega}{\mathrm{d} r} \simeq \frac{5 \Omega_{\odot}}{R_{\odot}} .
$$

Dicke's picture is that the angular velocity gradient is just slightly in excess of this marginally stable value, so that the turbulence it produces diffuses angular momentum outwards to balance the loss by the solar wind. If the typical turbulent velocity is $v$ and the type scale $l$ then

$$
4 \pi \rho r^{4}\langle v l\rangle \frac{\mathrm{d} \Omega}{\mathrm{d} r}=\frac{\mathrm{d} H}{\mathrm{~d} t}
$$

giving

$$
\langle v l\rangle \simeq 10^{4} \mathrm{~cm}^{2} \mathrm{~s}^{-1} .
$$

The diffusion coefficient $\langle v l\rangle$ determined by the angular momentum then determines the rate of depletion of lithium and if this thermally turbulent layer extends down to 0.56 of the solar radius, then the rate of depletion of lithium agrees tolerably well with the observations of the Hyades, Pleiades and the Sun.

We are therefore led to a picture in which the angular momentum of the core is either carried to the region of $0.56 R_{\odot}$ by magnetic stresses or is uncoupled from the rest of the star and is still rapidly spinning. This later is Dicke's own picture, but is incompatible with the new limits on the solar oblateness derived by Hill and Stebbins (1975). Their observations give a gravitational quadrupole moment

$$
J_{2} \leqslant \frac{1}{10} J_{2} \text { (Dicke) }
$$

where $J_{2}($ Dicke $)=2.5 \times 10^{-5}$ is the value predicted by Dicke's rapidly rotating core model. However, if the angular momentum of the core is carried outwards by magnetic stress so that $\Omega_{\text {core }} \simeq 2 \Omega_{\odot}$ then

$$
J_{2}=\frac{1}{100} J_{2}(\text { Dicke) }
$$

and is compatible with the Hill-Stebbins result.

But a number of questions immediately arise; why is the core of $0.56 R_{\odot}$ isolated from the rest of the Sun? Why does the magnetic field in the core, and indeed that in the envelope, not penetrate into the region $0.56<r / R_{\odot}<0.8$ ? At present no convincing model exists, but the success of Dicke's calculation in predicting the lithium depletion from the angular momentum loss suggests that the model is worth exploring. 
If this region of differential rotation exists, it is perhaps worth asking if the solar dynamo could operate there, since this is at least a region where the magnetic buoyancy time is of the order of years.

\section{Oscillations of the Sun}

Our understanding of the solar interior is even more confused by the recent observations of Hill et al. (1975) of solar oscillations. As part of their programme to determine the solar oblateness, they have discovered that the Sun is oscillating with frequencies in the range 0.5 to $4 \mathrm{MHz}$. The frequencies they observe fit tolerably well to the normal modes of oscillation of a detailed solar model calculated by Gough (1975). At present, it is not known whether these are radial or non-radial modes and the spectrum can be fitted with either, but as observations proceed, the nature of the oscillations will become clearer. Already they pose a problem for the theorist; what is the exciting mechanism? Could they be driven by the solar convective zone, or by a slowly mixing core, or are they indicators of the periodic instabilities proposed by Christensen-Dalsgaard et al. (1974)? In any case, do these waves contribute significantly to energy transport in the Sun, so that there is mechanical as well as radiative transport in the interior? Although the surface amplitudes are small $\delta R / R_{\odot} \simeq 10^{-6}$ this does not necessarily mean that the internal amplitudes are as small, and if a sufficiently large number of modes are excited even with small amplitude, they could carry a substantial fraction of the solar luminosity. Such mechanical transport has been suggested by Hoyle (1975) and Hill (1975) as being responsible for changing the internal structure of the Sun, and lowering the neutrino flux. While again no detailed models exist, this is an exciting possibility, and a rich field for future research.

\section{The Surface Convective Zone}

The convective theory used in modelling the Sun and solar-type stars is still the mixing length theory. In this 'theory' the convective heat flux $F_{c}$ is estimated by the expressions

$$
\begin{aligned}
& F_{c}=\left\langle c_{p} \rho v T\right\rangle=c_{p} \rho v \delta T \\
& v^{2}=\frac{g}{T} \delta T l, \quad \delta T=\Delta \nabla l
\end{aligned}
$$

where

$$
\Delta \nabla=\frac{\mathrm{d} T}{\mathrm{~d} r}-\left(1-\frac{1}{\gamma}\right) \frac{T}{P} \frac{\mathrm{d} P}{\mathrm{~d} r}
$$

and $l$ the mixing length is taken to be some multiple, usually one, of the pressure scale height $P /(\mathrm{d} P / \mathrm{d} r)$. As pointed out in Section 2 , the Sun provides no test of this theory since the observed solar radius is used to determine the mixing length!

Even for the Sun, the theory is clearly inadequate since it considers only motion on one scale $l$, whereas we observe the granulation, supergranulation and possibly giant 
cells; how can these be explained? One possibility is that the larger scales of motion represent a convective instability of the small scale equilibrium. To see how this could work, we note that the turbulent average of the heat equation gives a turbulent heat flux

$$
F_{c}=\kappa \nabla S \quad \kappa=\langle v l\rangle
$$

and a turbulent viscous transport

$$
\nu \nabla^{2} \mathbf{v} \quad \nu=\langle v l\rangle .
$$

The turbulent transport coefficients $\kappa$ and $\nu$ are then analogous to the kinetic transport coefficients $\kappa, \nu$, and so the mean flow equations can be unstable to convection just as the mean molecular equations can be unstable to convective motions. The transport coefficients are functions of depth $z$, so the mean flow Rayleigh number for the convective zone is

$$
R=\frac{g \alpha \beta d^{4}}{\kappa \nu}=R(z)
$$

If $R(z)$ is sufficiently large in the surface layers, these layers can be unstable and drive motions in the whole of the layer, whose characteristic scale is the depth of the unstable driving region. Calculations by myself and $\mathrm{G}$. Vickers for convection with varying transport coefficients show that this is a possibility in a Boussinesq fluid, and it is possible to show that the compressible case can be analysed in a similar way by a quasi-adiabatic approximation giving similar results (Roxburgh, 1975b). Indeed, it is possible that the giant cells represent a third scale in which the whole convective zone is unstable to giant cells influenced by rotation as in the Boussinesq calculations of Busse (1970), Durney (1975) and Gilman (1972, 1975), the mean flow equations and the quasi-adiabatic approximation yielding equations similar to those of their Boussinesq treatments (Roxburgh, unpublished).

But is the mixing length theory adequate to represent small scales? I believe the answer is no. The mixing length theory is a local order of magnitude theory and it could well be misleading. To see this we write down the full time independent energy equation for a gas in the form

$$
\operatorname{div}\left[\frac{\gamma}{\gamma-1} P \mathbf{v}+\frac{1}{2} \rho \mathbf{v} v^{2}+F_{R}\right]=0
$$

The mixing length theory is obtained by using a turbulent decomposition

$$
\rho=\rho_{1}+\rho_{1}, \quad T=T_{0}+T_{1}, \quad v=v_{1}
$$

such that

$$
\langle\rho v\rangle=0 \quad\left\langle v_{1}\right\rangle=-\left\langle\rho_{1} v_{1}\right\rangle / \rho_{0}
$$

The pressure fluctuations are then taken to be negligible so

$$
\left\langle\frac{P_{1}^{2}}{P_{0}^{2}}\right\rangle \ll\left\langle\frac{\rho_{1}^{2}}{\rho_{0}^{2}}\right\rangle,\left\langle\frac{T_{1}^{2}}{T_{0}^{2}}\right\rangle, \quad \frac{\rho_{1}}{\rho_{0}}=-\frac{T_{1}}{T_{0}}
$$


and then neglecting $\left\langle\rho v^{2} v\right\rangle$ so that

$$
\begin{aligned}
F_{c}=\frac{\gamma}{\gamma-1}\langle p v\rangle+\frac{1}{2}\left\langle\rho v v^{2}\right\rangle & \simeq \frac{\gamma}{\gamma-1} P_{0}\left\langle v_{1}\right\rangle=-\frac{\gamma}{\gamma-1} P_{0} \frac{\left\langle\rho_{1} v_{1}\right\rangle}{\rho_{0}} \\
& =\frac{\gamma}{\gamma-1} \frac{P_{0}}{T_{0}}\left\langle T_{1} v_{1}\right\rangle=c_{p} \rho_{0} v_{1} T_{1} .
\end{aligned}
$$

Then on using

$$
T_{1}=\Delta \nabla l, \quad \text { and } \quad v_{1}^{2}=\frac{g}{T} T_{1} l
$$

the standard expressions follow.

However, this is clearly inconsistent since if $\left\langle P_{1}^{2} / P_{0}^{2}\right\rangle$ can be neglected, then on taking the scalar product of the momentum equation with $\mathbf{v}$ and averaging we find

$$
\langle v\rangle \frac{\mathrm{d} P_{0}}{\mathrm{~d} z}=-\frac{\mathrm{d}}{\mathrm{d} z}\left\langle\frac{\rho \mathbf{v} v^{2}}{z}\right\rangle
$$

so that

$$
F_{E}=\frac{\gamma}{\gamma-1}\langle P v\rangle=\frac{c_{p} T}{g} \frac{d}{\mathrm{~d} z}\left(\frac{1}{2} \rho \mathbf{v} v^{2}\right) \equiv \frac{c_{P} T}{g} \frac{\mathrm{d} F_{k}}{\mathrm{~d} z}
$$

which is of the same order as the kinetic energy flux $\left\langle\frac{1}{2} \rho \mathbf{v} v^{2}\right\rangle$. Indeed in terms of the total flux $F$ the energy equation has the integral

$$
F_{\kappa}=p^{1-1 / \gamma} \int \frac{g}{c_{p} T} p^{1 / \gamma-1}\left(F-F_{R}\right) \mathrm{d} z
$$

so that $F_{k}$ cannot be zero at the boundaries where $F=F_{R}$, nor is $F_{E}$, it is only the sum that vanishes. In this case there has to be substantial overshooting into the stable region where $F-F_{R}$ is negative and we need a non-local theory of convection. Preliminary calculations by the author suggest that this region can be as large as a scale height at the bottom of the convective layer, and about 0.3 of a scale height at the top (Roxburgh, 1975c).

However, it is clear that such a model is not correct either since the pressure fluctuations cannot be neglected; they are necessary both to stop the vertical motions and to drive the horizontal motions and must, therefore, be comparable to the density and temperature fluctuations. In a correct theory, we need to include the correlations $\left\langle P_{1} v_{1}\right\rangle$ and have a third term in the expression for the energy flux so that

$$
F_{c}=\frac{\gamma}{\gamma-1}\left\langle P_{0} v_{1}\right\rangle+\frac{\gamma}{\gamma-1}\left\langle P_{1} v_{1}\right\rangle+\frac{1}{2}\left\langle\rho v v^{2}\right\rangle=F=F_{R}
$$

but the essential part of the previous result remains that $F-F_{R}$ can be zero without each term in the expression for $F_{c}$ vanishing. Convective overshooting will still be important.

Clearly much remains to be done before any adequate theory of turbulent convection is available. 


\section{The Solar Interior}

What then is the Sun like? We may conjecture a model consisting of:

(a) A core of $\sim 0.56 R_{\odot}$ that is slowly but steadily mixed, the mixing currents driven by the ${ }^{3} \mathrm{He}$ instability.

(b) Energy transport by waves excited by the mixing core.

(c) A trapped magnetic field in the central core.

(d) An intermediate layer of weak thermally driven turbulence between $0.56 R_{\odot}$ and $0.7 \boldsymbol{R}_{\odot}$ diffusing both angular momentum and lithium depleted matter.

(e) A convective overshoot region between $0.7 R_{\odot}$ and $0.8 R_{\odot}$.

(f) The convective zone proper between $0.8 R_{\odot}$ and $1 R_{\odot}$.

(g) A convective overshoot region into the photosphere of order $100 \mathrm{~km}$.

(h) The central core having an angular velocity $\sim 2 \Omega_{0}$.

Such a model is highly speculative!

\section{Conclusion}

In conclusion let me emphasise that as yet we have no satisfactory model of the internal structure of the Sun and solar type stars. If the central regions are mixed, this will have a sigfnificant effect on the evolution of stars, on estimates of the age of globular clusters, the evolution of galaxies, the determination of the deceleration parameter in cosmology and so on the determination of the structure of the Universe in which we live. We no longer believe that the Sun is the centre of the Universe, but the solution of the puzzles posed by the Sun is central to our understanding of stellar evolution and may well influence the picture we have of the Universe we live in.

\section{References}

Busse, F. H.: 1970 Astrophys. J. 159, 629.

Christensen-Dalsgaard, J., Dilke, F. W. W., and Gough, D. O.: 1974, Monthly Notices Roy. Astron. Soc. $169,429$.

Davis, R.: Bull. Amer. Phys. Soc. 17, 527.

Dicke, R. H.: 1972, Astrophys. J. 171, 331.

Dicke, R. H. and Goldenberg, H. M.: 1967 Phys. Rev. Letters 18, 313.

Dicke, R. H. and Goldenberg, H. M.: 1974 Astrophys. J. Suppl. 27, 131.

Dilke, F. W. W. and Gough, D. O.: 1972, Nature 240, 262.

Dirac, P. A. M.: 1938, Proc. Royal Soc. A, 165, 199.

Durney, B. R.: 1971, Astrophys. J. 163, 353.

Durney, B. R.: 1972, Proceedings of the 1971 Asilomar Conference on the Solar Wind, p. 282 NASA publ.

Durney, B. R.: 1975, this volume, p. 243.

Fowler, W. A.: 1972, Nature 238, 24.

Fricke, K.: 1968 Z. Astrophysik 68, 317.

Goldreich, P. and Schubert, G.: 1967, Astrophys. J. 150, 571.

Gilman, P. A.: 1972, Solar Phys. 27, 3.

Gilman, P. A.: 1975, this volume, p. 207.

Hayashi, C.: 1961, Proc. Astron. Soc. Japan 13, 450.

Hayes, J.: 1975, Proceedings of the Solar Constant Workshop (in press).

Hill, H. A. and Stebbins, R. T.: 1975, Ann. New York Acad. Sci. (in press).

Hill, H. A., Stebbins, R. T., and Brown, T. M.: SCLERA Report 1975.

Hill, H. A., and McCullen, J. D., Brown, T. M., and Stebbins, R. T.: SCLERA Report 1975. 
Hoyle, F.: 1975 Astrophys. J. 197, L127.

Milne, E. A.: 1935, Gravitation and World Structure, Oxford University Press.

Roxburgh, I. W.: 1964, Monthly Notices Roy. Astron. Soc. 128, 157.

Roxburgh, I. W.: 1973 (unpublished note).

Roxburgh, I. W.: 1974, Nature 248, 209.

Roxburgh, I. W.: 1975a, Monthly Notices Roy. Astron. Soc. 170, 35p.

Roxburgh, I. W.: 1975b (to be published).

Roxburgh, I. W.: 1975c (to be published).

Schwarzschild, M.: 1968 Structure and Evolution of the Stars, Princeton Univ. Press.

Shaviv, G. and Buchall, J. N.: 1969 Astrophys. J. 155, 135.

Skumanich, A.: 1972, Astrophys. J. 171, 565.

Ulrich, R. K.: 1975 (to be published).

Wallerstein, G. and Conti, P.: 1969, Ann. Rev. Astron. Astrophys. 99.

Wilson, O. C.: 1963, Astrophys. J. 140, 1401.

\section{DISCUSSION}

Parker: There are two comments that come to mind in your review of possible solutions to the dilemma presented by the Sun. One is that any non-uniform rotation participating in the solar dynamo must be driven by thermal convective effects. There is not enough energy in solar rotation to drive the present dynamo for the life of the Sun. Second, the fields that might be trapped in the central core of the Sun cannot be very strong, because very strong fields are lost by magnetic buoyancy. For instance, the $10^{7} \mathrm{G}$ that some have postulated to resolve the neutrino dilemma would be lost in a period of the order of only $10^{1} \mathrm{yr}$.

Mestel: I am certainly not against primeval magnetic fields, and in fact think it quite likely that beneath the solar convection zone there is zone trapped flux (quite distinct from the solar dynamo field). However, I doubt if it would stay confined to your central region: I would expect it to diffuse so as to couple the base of the convection zone with the core, so probably wiping out your $\Omega$ gradient. There are other dynamical consequences of a primeval magnetic field which may be relevant. Unless such a field has a high degree of symmetry with respect to the rotation axis it tends to set up oscillatory rotations, with the period long compared with the rotation period but short compared with the thermal time scale. The amplitude of these rotations is small, but they may still lead by virtue of finite resistivity to a steady diffusion of material that would be significant within the leisurely time-scale of stellar evolution. Conceivably this could be relevant to both the neutrino and lithium problems. To Dr Parker's remarks on magnetic buoyancy: in a radiative zone the rate at which flux tubes rise is determined by heat-flow essentially an Eddington-Sweet effect; and the motion may very well be suppressed altogether by a slight gradient of mean molecular weight. Of course, all these remarks presume the hypothetical primeval field has a dynamically stable structure - e.g. that it has toroidal flux tubes linking the poloidal loops.

Gilman: The suggestion that there could be dynamo action in the overshoot region below the convection zone is interesting, but if the rotation rate increases as fast with depth there, as you suggest, we should expect to see large scale magnetic features at surface rotating much faster than they do.

Weiss: You quoted the generally accepted result that the Sun was fully convective before approaching the main sequence. This was found by Hayashi but Larson, starting from different initial conditions, finds that only outer $50 \%$ by radius was convective. So we have yet another uncertainty. Moreover the central magnetic field may remain distinct from that in the outer convective zone. 